\title{
Amputación corporal por accidente de trabajo en auxiliar de enfermería
}

\author{
Severed body segment of nursing assitant at workplace
}

\author{
Alexander Finol Muñoz', Gloria Ortega Marín', Julián Domínguez Fernández ${ }^{1,2}$, Johanna Rivero Colina', Manuel \\ Usero Fernández', Miguel Espejo García'
}

1. Hospital Universitario de Ceuta. España.

2. Unidad Docente Multiprofesional de Salud Laboral. Hospital Universitario de Ceuta. España.

\section{Recibido: 09-08-14}

Aceptado: 03-11-14

\author{
Correspondencia \\ Alexander José Finol Muñoz. \\ Hospital Universitario de Ceuta \\ Loma Colmenar $\mathrm{s} / \mathrm{n}$. $^{\circ}$ \\ Edificio 2. Planta-1. \\ 51003. Ceuta. España \\ Correo electrónico: finolito@hotmail.com
}

Resumen

Los auxiliares de enfermería son un rango profesional expuesto a múltiples riesgos por las actividades inherentes a su trabajo, expuestos constantemente a sustancias desinfectantes que sin el uso apropiado de equipos de protección individual, puede provocar efectos adversos y lesiones en el trabajador. Caso Clínico: Mujer de 51 años de edad, auxiliar de enfermería, con antecedentes de Diabetes Mellitus tipo I y Síndrome de Túnel Carpiano. Presenta derrame accidental de líquido mientras llenaba envase de Biguanid®, cayéndole en todo el cuerpo, por lo que decide cambiarse el uniforme entero, conservando calcetines y zapatos por el resto del turno. Posteriormente presenta lesiones en región dorsal de $4 \mathrm{t}^{\circ}$ dedo de pie izquierdo, las cuales reciben tratamiento médico y seguimiento, con evolución tórpida, se evidencia edema y osteomielitis de la falange por lo que se decide amputar el dedo afectado. Una vez recuperada, fue estudiada con pruebas de provocación, evidenciando la susceptibilidad de la trabajadora a dicho desinfectante. Se propone al Instituto Nacional de Seguridad Social (INSS) como accidente de trabajo y una indemnización por lesión permanente no invalidante, ambas peticiones con respuesta favorable para la trabajadora. Actualmente sigue desempeñando sus funciones como auxiliar en el hospital. El cumplimiento y vigilancia de las normas de prevención, basados en los riesgos laborales permitirá evitar este tipo de incidentes en la población laboral, evitando a largo plazo lesiones corporales, discapacidades y bajas laborales que alteran la calidad de vida del trabajador y de su entorno profesional.

Med Segur Trab (Internet) 2014; 60 (237) 786-793

Palabras clave: Orbicular, músico, Satchmo.

Abstract

Nursing assistants are a professional rank exposed to multiple risks by the inherent activities to their work. They are constantly exposed to disinfectant substances that can cause adverse effects and injuries to the worker unless they use the appropriate personal protective equipment. Clinical case: 51-year-old woman, 
nursing assistant, with type I Diabetes Mellitus and Carpal Tunnel Syndrome history. She accidentally spilled liquid on her body while filling a package of Biguanid®. Consequently she decides to change the whole uniform, keeping socks and shoes during the rest of the turn. Later on she presents injuries in the dorsum of the fourth left finger, which received medical treatment and monitoring of slow evolution. The affected finger is amputated as oedema and osteomyelitis of the phalanx are evident. Once recovered, she was studied with provocation tests demonstrating the susceptibility of the worker to the disinfectant. A compensation by permanent non-invalidating injuries and work accident was requested to the National Social Security Institute (NSSI) with favourable answer for both requests. She still executes her functions as an assistant in the hospital. The compliance and monitoring of the norms of the labour risks prevention will allow to avoid this kind of incidents in the labour population, avoiding long-term corporal injuries, labour inabilities and sick leaves that modify the quality life of the worker and their professional surroundings.

Med Segur Trab (Internet) 2014; 60 (237) 786-793

Keywords: Amputation, work accident, nursing assistant, Diabetes Mellitus. 


\section{INTRODUCCIÓN}

Por las funciones propias del auxiliar de enfermería, este rango profesional se encuentra expuesto a múltiples riesgos durante el desempeño de sus tareas, tales como el aseo y rasurado de pacientes, limpieza de materiales, recepción y entrega de comidas, reposición de materiales (batas, sabanas, toallas, gasas, guantes, etc.) funciones que facilitan la faena de enfermeras y médicos con el fin de garantizar una atención integral del paciente que lo amerita según su estado de salud ${ }^{1}$.

A su vez, se encuentran en contacto permanente con productos químicos desinfectantes de uso común en instituciones de salud, que presentan altas demandas higiénicas por la presencia de múltiples agentes virales, bacterianos y fúngicos. Dentro de sus componentes, se encuentran sustancias que alteran la integridad de la salud de los auxiliares de enfermería si no se toman las medidas adecuadas de prevención en su manejo diario.

El contacto prolongado con este tipo de sustancias desinfectantes, que incluyen potentes sustancias químicas antibacterianas de amplio espectro, puede provocar lesiones en la piel, que dependiendo de la susceptibilidad del potencial usuario puede acarrear lesiones permanentes en la piel, incluso mutilaciones corporales.

Dentro de los más usados en el Hospital Universitario de Ceuta, se encuentra el BIGUANID®, un líquido de apariencia clara, color azul-verdoso con PH: Sin diluir 8.5 +/- 0.5. Su Composición: alkyldimethylbenzylammoniumchloride, alcylpropylendiamin1,5-bis-guanidinium acetato polihexanide, sin aldehidos ni fenoles, validado por la Comunidad Europea, con propiedades fungicidas, bactericidas y virucida frente a los patógenos más comunes. Sus campos de aplicación incluye, limpieza y desinfección de aparatos y utensilios médicos, también compatible para la limpieza de suelos, con niveles de desinfección variables según la concentración y el tiempo de aplicación ${ }^{2}$.

El caso clínico que se describe a continuación, plantea la importancia del uso de equipos de protección individual, el correcto manejo de este tipo de sustancias desinfectantes y el grado de afectación corporal que puede sufrir la persona, tomando en consideración la susceptibilidad individual; factor fundamental en la evaluación personal de los trabajadores en relación con la tarea que debe cumplir.

\section{CASO CLÍNICO}

Mujer de 51 años de edad, auxiliar de enfermería, con antecedentes de Diabetes Mellitus tipo I y Síndrome de Túnel Carpiano. El día 20/02/2011, durante jornada de trabajo en planta, presenta derrame accidental de líquido mientras llenaba envase de Biguanid®, cayéndole en todo el cuerpo, por lo que decide cambiarse el uniforme entero, conservando calcetines y zapatos por el resto del turno de trabajo. Posteriormente presenta dos lesiones ampollosas en región dorsal de $4 \mathrm{t}^{\circ}$ dedo de pie izquierdo, las cuales son tratadas y seguidas por cirugía general. Comienzan a aparecer signos de flogosis y secreción (Ver imágenes 1, 2), la cual se cultiva resultando crecimiento de Staphylococcus Aureus meticilín-resistente, indicándose tratamiento según antibiograma. A pesar de las medidas se complica con edema y osteomielitis de la falange (ver imagen 3) por lo que se decide amputar el dedo afectado. Una vez recuperada y con el estatus de incapacidad transitoria, fue estudiada con pruebas de provocación, evidenciando la susceptibilidad de la trabajadora a dicho desinfectante (ver imagen 4). Se propone al órgano competente Instituto Nacional de Seguridad Social (INSS) como accidente de trabajo y una indemnización por lesión permanente no invalidante. Actualmente sigue desempeñando sus funciones como auxiliar en el hospital. 
Imagen 1

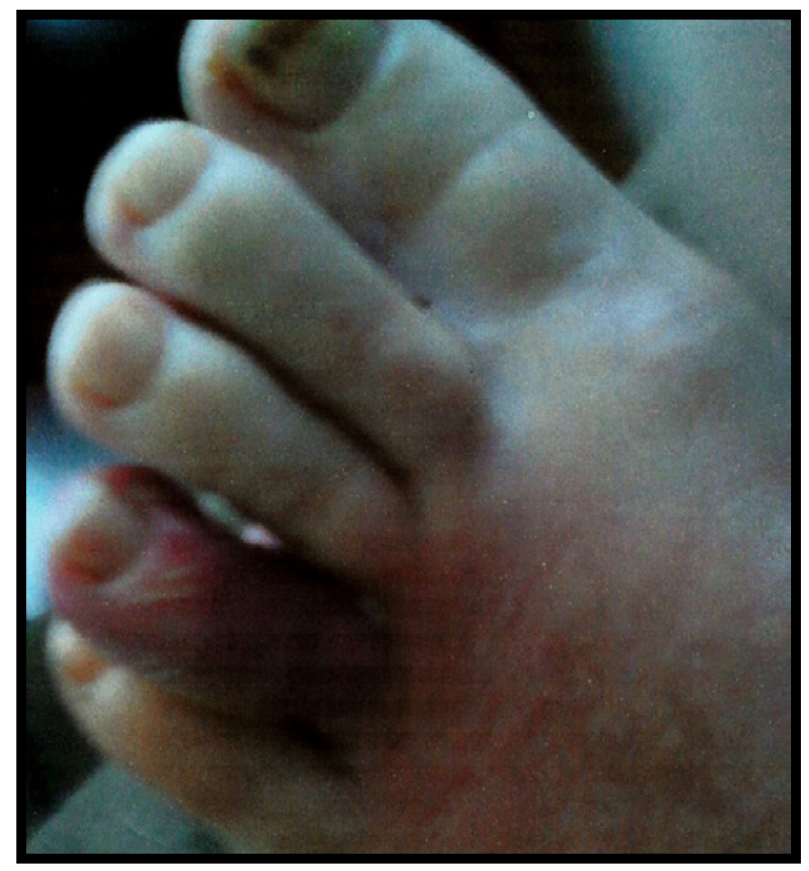

Imagen 2

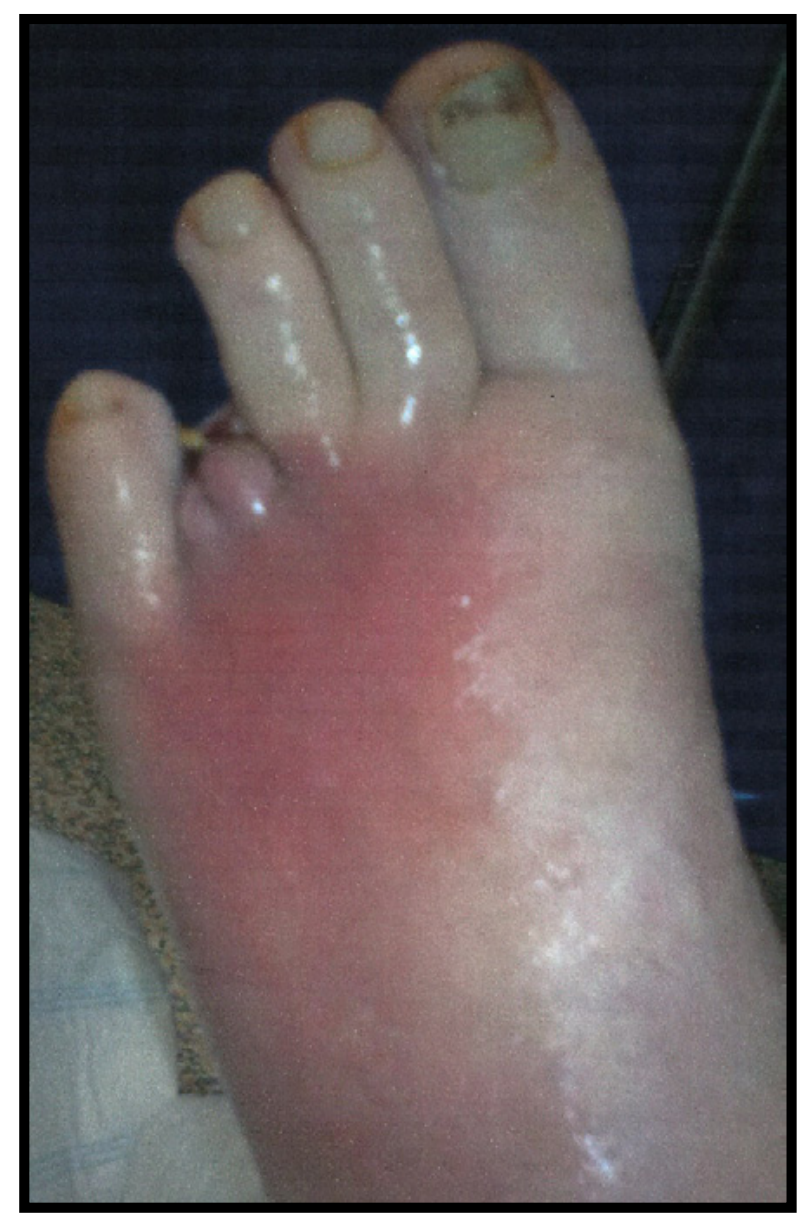




\section{Imagen 3}

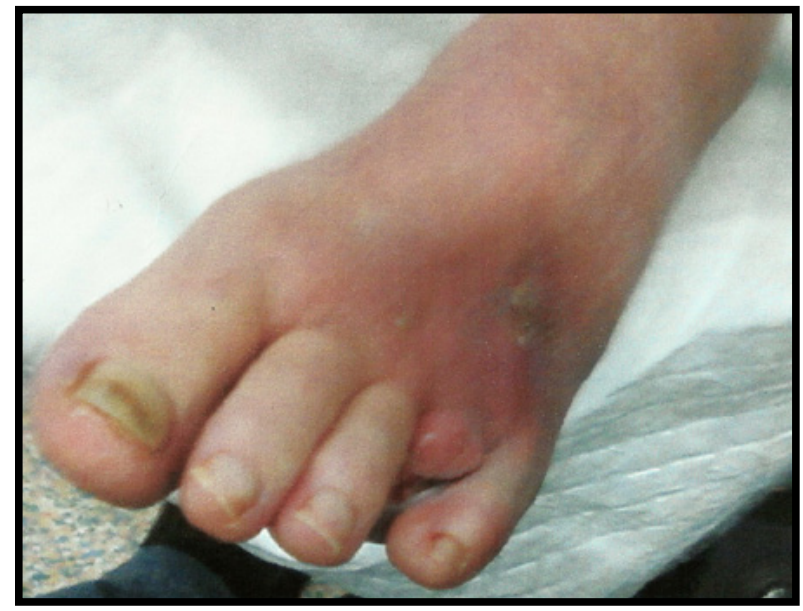

Imagen 4

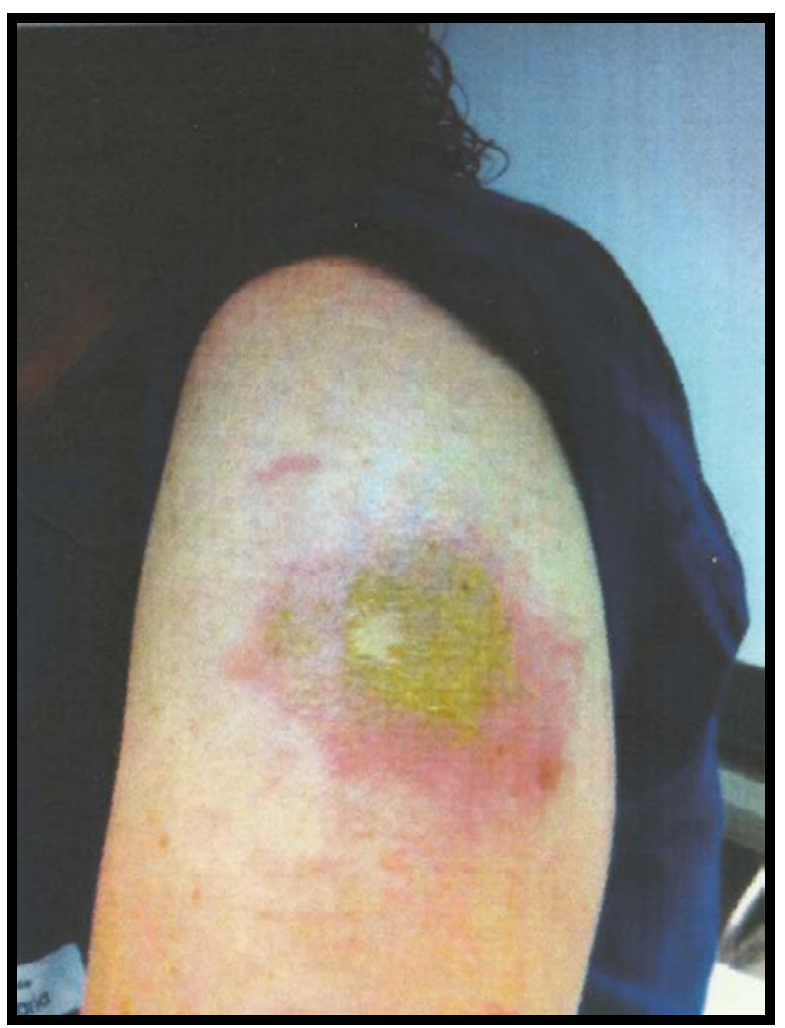

* Imagenes tomadas de la Historia Clínica de la Trabajadora

\section{DISCUSIÓN Y CONCLUSIONES}

En el mundo hay más de 347 millones de personas con diabetes. La diabetes es una enfermedad crónica que aparece cuando el páncreas es incapaz de producir suficiente insulina o cuando el organismo no utiliza eficazmente la insulina que produce. La insulina es una hormona que regula el azúcar en la sangre. El efecto de la diabetes no controlada es la hiperglucemia, que con el tiempo daña gravemente muchos órganos y sistemas, especialmente los nervios y los vasos sanguíneos. La neuropatía diabética, combinada con la reducción del flujo sanguíneo por los daños en la micro y macro circulación 
sanguínea, incrementan el riesgo de úlceras de los pies y en última instancia, amputación. Otras complicaciones frecuentes son la retinopatía y nefropatía diabética. La trabajadora descrita en el caso tiene como antecedente importante Diabetes Mellitus tipo I, lo que la predispone a presentar todas las complicaciones antes mencionadas, con suma importancia la afectación neurológica y circulatoria, que podría justificar la evolución tórpida que presentó posterior al contacto con el líquido desinfectante ${ }^{3}$.

La piel, como vía de entrada de agentes químicos, es una barrera que presenta una enorme cantidad de singularidades que determinan, en gran medida, la mayor o menor velocidad de entrada de cada especie química. Una descripción detallada de la estructura de la piel y de los mecanismos de penetración percutánea en función de la naturaleza del agente químico, del medio en el que va disperso, del estado de la piel, entre otros, permitirá predecir el efecto final de dicha exposición. Los efectos derivados de la exposición dérmica a sustancias químicas pueden ser locales, provocando trastornos en la piel, tales como irritaciones, dermatitis, sensibilización o sistémicos, causando alteraciones o daños en órganos o sistemas específicos. Esta trabajadora por los antecedentes mencionados y por su sensibilidad al producto utilizado, ha terminado con una amputación de la parte que permaneció con mayor tiempo expuesta al Biguanid® ${ }^{4}$.

Dentro de los riesgos por manejo de químicos en el lugar de trabajo tenemos la absorción a través de la piel, ingestión, contacto de la piel o los ojos con el agente químico, entre otros, que sumado a los factores de riesgo determinados por la gestión incorrecta de EPI (Equipos de protección individual), procedimiento de trabajo inadecuado, existencia de medios de control de fugas y derrames, envases inadecuados y sistema de trasvase incorrecto. Analizando el caso con detenimiento, la trabajadora ha incurrido en varios fallos al realizar el trasvase del líquido del desinfectante, el uso incorrecto de EPI y normas básicas de protección frente a derrame de líquidos, como el lavado con agua abundante y cambio de ropa, lo que evidentemente ha desencadenado toda esta serie de eventos desafortunados para su salud, allí radica la importancia de cumplir con las medidas preventivas según la evaluación de riesgos inherente a cada actividad laboral, ya que han sido implantadas con la finalidad de eliminar o reducir un riesgo determinado 5 .

Es necesario descartar que las lesiones ocurridas en la trabajadora fueran consecuencia de otra sustancia de uso común, ya que son muy frecuentes este tipo de reacciones frente a conservantes de jabones, algunas fragancias, metales diversos, bisutería, entre otros, pudiendo ser confundidos según el tipo de reacción con la piel. Con el fin de comprobar la relación entre la exposición al líquido desinfectante y la amputación posterior al accidente laboral declarado, se decidió realizar las respectivas pruebas epicutáneas (pruebas del parche) para confirmar la sensibilización a alguno de los componentes del Biguanid® (Ver Imagen 4). Fue evidente y clara la respuesta a la prueba. Se puede afirmar la susceptibilidad de la trabajadora con el producto, donde juega un papel fundamental el tiempo de exposición y su patología de base.

El Accidente de Trabajo, desde la perspectiva legal, queda definido como toda lesión corporal que el trabajador sufra como consecuencia del trabajo que ejecute por cuenta ajena $^{6}$. Así mismo se ha establecido en la Ley General de la Seguridad Social que las lesiones, mutilaciones y deformidades de carácter definitivo, causadas por accidentes de trabajo y enfermedades profesionales serán indemnizadas ${ }^{7}$. A la trabajadora se le ha reconocido una indemnización por lesión permanente no invalidante. Al finalizar su recuperación, se ha reintegrado al puesto de trabajo habitual.

El objetivo de la medicina del trabajo, es garantizar la vigilancia de la salud individual y colectiva de los trabajadores. Mediante la evaluación de riesgo de los puestos de trabajo, se pueden crear ciertas medidas preventivas que en función de su cumplimiento podrán disminuir incluso evitar los accidentes laborales. El empresario está obligado a asumir ese compromiso. El principal propósito de la vigilancia de la salud va a ser comprender mejor el impacto que el trabajo tiene sobre la salud de los trabajadores, de tal forma que genere información que oriente a la toma de decisiones para mejorar las condiciones de trabajo5. 
Por otra parte, la vigilancia debe posibilitar que se identifiquen, tan pronto como sea posible, los efectos adversos sobre el bienestar físico y mental, de tal manera que se pueda evitar la progresión hacia un ulterior daño para la salud más importante. Recalcar en la población de trabajadores el cumplimiento de las normas de prevención de riesgos laborales, incidirá directamente en su propia salud, evitando discapacidades y trastornos que afecten su vida cotidiana.

No es éticamente correcto, adjudicar las fallas en la actuación en temas de prevención exclusivamente a nuestros trabajadores, por lo que se debe asumir parte de la responsabilidad en el asunto.

Desde el punto de vista práctico, el diseño e incorporación de nuevas tecnologías en el manejo de productos químicos, podría disminuir la incidencia de este tipo de accidente de trabajo, como por ejemplo:

1. Mejorando la ergonomía de los envases (sistemas anti-derrame, asas de agarre amplio, superficies corrugadas).

2. Uso de envases desechables con reemplazo del recipiente completo (evita la necesidad de relleno de líquidos).

3. Equipos impermeables que no dificulten la respiración de la piel, motivo que en la mayoría de los casos hacen que los trabajadores hagan uso incorrecto de los mismos o peor aún, prescindan de ellos.

Antes de contratar un trabajador o de cambiarlo de puesto de trabajo, debería ser sometido a un examen de salud con el objetivo de determinar su aptitud para ocupar el nuevo puesto, que supondrá una determinada exposición a ciertos agentes químicos frente a los que el trabajador puede ser especialmente sensible o que pueden agravar una dolencia ya existente, en cuyo caso habrá que hacer énfasis en la formación contínua en materia de prevención, vigilancia exhaustiva del cumplimiento de dichas normas y facilitar los equipos de prevención para su uso ${ }^{5}$.

La finalidad de los servicios de prevención no puede estar basada exclusivamente en la dotación de EPI, debe enfocarse mucho más en la formación y vigilancia en el cumplimiento de las normas de prevención. Su función no termina allí, dichos servicios deben lograr incorporar dentro del esquema de trabajo habitual de sus trabajadores, el cumplimiento de los reglamentos de protección, haciéndolos parte de la rutina laboral y no un requisito para acatar solo bajo observación de algún superior. Tener esta visión dentro del SPRL, es fundamental para unir esfuerzos en el logro de dichas metas.

De la misma forma, disponer de algún tipo de protocolo de actuación especifico según las patologías que puedan derivar un riesgo agregado al desenvolvimiento de ese trabajador frente a la exposición a agentes químicos diversos, podría incorporarse una evaluación más cercana, jornadas de formación obligatoria con intervalos cortos de tiempo entre sesiones, evaluación contínua del cumplimiento de dichas normas, designando un personal especial para vigilar su cumplimiento, sistemas de evaluación de gestión de calidad que aportarán información sobre el feedback de los trabajadores hacia el servicio de prevención, programas de reconocimiento al mérito o algún tipo de recompensa por correcto cumplimiento de las normas, aspectos que refuercen las conductas positivas y evitan desenlaces trágicos como el relatado en este caso clínico.

\section{BIBLIOGRAFÍA}

1. Funciones del auxiliar de enfermería. Disponible en: http://www.auxiliar-enfermeria.com/funciones_ auxenf.htm.

2. Biguanid. Especificaciones Técnicas. Disponible en: file://C:/Users/pc\%20admin/Downloads/184613006 6533bb1201e4e9.pdf

3. Diabetes. Organización Mundial de la Salud. Disponible en: http://www.who.int/mediacentre/factsheets/ fs312/es/. 
4. Salazar L, Heras F, Maqueda J. Directrices para la decisión clínica en enfermedades profesionales. Ministerio de Economía y Competitividad. Instituto Nacional de Seguridad e Higiene en el Trabajo. Disponible en: http://www.insht.es/InshtWeb/Contenidos/Documentacion/FICHAS\%20DE\%20 PUBLICACIONES/EN\%20CATALOGO/VIGILANCIA\%20DE\%20LA\%20SALUD/Directrices\%20para\%20 la\%20toma\%20de\%20decisiones/cancer_cutaneo.pdf.

5. Guía Técnica para la evaluación y prevención de los riesgos relacionados con el uso de agentes químicos presentes en los lugares de trabajo. Instituto Nacional de Seguridad e Higiene en el Trabajo (INSHT). Disponible en: http://www.insht.es/InshtWeb/Contenidos/Normativa/GuiasTecnicas/Ficheros/g_AQ.pdf.

6. Ministerio de Empleo y Seguridad Social. Real Decreto Legislativo 1/1994, de 20 de junio, por el que se aprueba el Texto Refundido de la Ley General de la Seguridad Social. Disponible en: http://www.segsocial.es/Internet_1/Normativa/095093?ssSourceNodeId=1139\#A115

7. Gobierno de España. Ministerio de Empleo y Seguridad Social. Disponible en: http://www.seg-social.es/ Internet_1/Masinformacion/Tramitesy Gestiones/ Prestacion por lesion 045997/index.htm.

8. Ciércoles J, Castro J. Reimplante de miembro superior. Inserción al puesto de trabajo tras accidente laboral. Revista Enfermería del Trabajo. 2014; 4: 71-75.

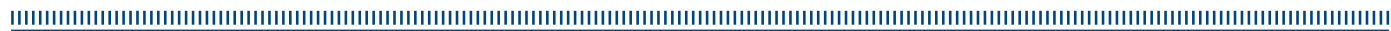

